\title{
A NEW APPROACH TO PÓLYA URN SCHEMES AND ITS INFINITE COLOR GENERALIZATION
}

\author{
ANTAR BANDYOPADHYAY AND DEBLEENA THACKER
}

\begin{abstract}
In this work we generalize Pólya urn schemes with possibly infinitely many colors and extend the earlier models described in [4, 5, 7]. We provide a novel and unique approach of representing the observed sequence of colors in terms a branching Markov chain on random recursion tree. This enables us to derive fairly general asymptotic for our urn schemes. We then illustrate through several examples that our method can easily derive the classical results for finite urns, as well as, many new results for infinite color urns.
\end{abstract}

\section{INTRODUCTION}

In recent days various urn schemes and their many generalizations have been a key element of study for random processes with reinforcements [3 $[5,8,11,13,14$, 16, 18, 24, 25, 27, 29]. Starting from the seminal work by Pólya [30], various types of urn schemes with finitely many colors have been widely studied in literature 11,3, 8, 9, 11, 12, 16, 18, 20, 22, 24, 28, 29, 29] provides an extensive survey of the known results. However, other than the classical work by Blackwell and MacQueen 7], there has not been much development of infinite color generalization of the Pólya urn scheme. Recently the authors studied a specific class of urn models with infinitely many colors where the color set is indexed by the $d$-dimensional integer lattice $\mathbb{Z}^{d}, 4,[5$. These works nicely complement the work [7] by introducing examples of infinite color schemes with "off-diagonal" entries and showed that the asymptotic behavior is essentially determined by an underlying random walk.

In this paper, we further generalize urn schemes with colors index by an arbitrary set $S$ endowed with a $\sigma$-algebra $\mathcal{S}$. As we will see in the sequel, the classical models can be realized as a sub-model when $S$ is finite and in that case $\mathcal{S}$ will simply be the power set of $S$, which we will denote by $\wp(S)$. The non-classical case discussed in [7] can also be obtained by appropriately choosing the measurable space $(S, \mathcal{S})$ as the Borel space of a Polish space $S$. Further the models described in [4, 5] can be obtained by choosing $S=\mathbb{Z}^{d}$ and $\mathcal{S}=\wp\left(\mathbb{Z}^{d}\right)$.

However, it is worthwhile to note that we will only consider balanced urn schemes. For $S$ countable (finite or infinite), it means that if $R:=((R(i, j)))_{i, j \in S}$ denotes the replacement matrix, that is, $R(i, j) \geq 0$ is the number of balls of color $j$ to be placed in the the urn when the color of the selected ball is $i$, then for a balanced urn, all row sums of $R$ are constant. In this case, without loss of any generality,

2010 Mathematics Subject Classification. Primary: 60F05, 60F10; Secondary: 60G50.

Key words and phrases. infinite color urn, reinforcement processes, urn models, embedding theorem, irreducible, aperiodic positive recurrent matrices, random recursive trees, branching random walks, branching Markov chains, random replacement matrices. 
it is somewhat customary to assume that that $R$ is a stochastic matrix [8, 9]. For more general $S$ we refer to the next subsection for further details.

The main contribution of this work is two "representations" of the urn model with a Markov chain on $S$, which we will call the associated Markov chain. These representations are novel and useful in deriving asymptotic results for the expected and random configurations of the urn.

There are few standard methods for analyzing finite color urn models which are mainly based on martingale techniques [8, 9, 16, 22, stochastic approximations [26] and embedding into continuous time pure birth processes [1, 3, 23, 24. Typically the analysis of a finite color urn is heavily dependent on the Perron-Frobenius theory 31 of matrices with positive entries and Jordon Decomposition 15] of finite dimensional matrices [1, 3, 8, 16, 22, 24]. The absence of such a theory when $S$ is infinite, makes the analysis of urn with infinitely many colors quite difficult and challenging. Instead in [7] the results were derived using exchangeability of the observed sequence of colors. However, as observed in [5], exchangeability fails in the presence of off-diagonal entries and in [5, the authors took a different approach of marginally embedding the observed sequence of colors to the underlying random walk sequence. The major contribution of this work is to further this embedding for any general urn scheme and derive asymptotic results by bypassing the standard martingale and matrix theoretic techniques.

1.1. Model. We consider the following generalization of Pólya urn scheme where the colors are indexed by a non-empty subset $S$ of $\mathbb{R}^{d}$ for some $d \geq 1$, such that, under subspace topology $S$ is a Polish space. A necessary and sufficient for $S$ to be Polish is that it is a $G_{\delta}$-set, that is, $S$ is a countable intersection of open sets, 10. We endow $S$ with the corresponding Borel $\sigma$-algebra and denote it by $\mathcal{S}$. Let $\mathcal{M}(S)$ and $\mathcal{P}(S)$ denote respectively the set of all finite measures and the set of all probability measures on the measurable space $(S, \mathcal{S})$. Note that the classical case when $S$ is finite or the non-classical cases discussed in 4, 5] are obtained by taking $S$ a discrete subset of $\mathbb{R}^{d}$ of appropriate cardinality.

Let $R: S \times \mathcal{S} \rightarrow[0,1]$ be a Markov kernel on $S$, that is, for every $s \in S$, as a set-function of $\mathcal{S}, R(s, \cdot)$ is a probability measure on $(S, \mathcal{S})$; and for every $A \in \mathcal{S}$, the function $s \mapsto R(s, A)$ is $\mathcal{S} / \mathcal{B}_{[0,1]}$-measurable.

By a configuration of the urn at time $n \geq 0$, we will consider a finite measure $U_{n} \in \mathcal{M}(S)$, such that, if $Z_{n}$ represents the randomly chosen color at the $(n+1)$-th draw then the conditional distribution of $Z_{n}$ given the "past", is given by

$$
\mathbb{P}\left(Z_{n} \in d s \mid U_{n}, U_{n-1}, \cdots, U_{0}\right) \propto U_{n}(d s) .
$$

Formally, starting with $U_{0} \in \mathcal{P}(S)$ we define $\left(U_{n}\right)_{n \geq 0} \subseteq \mathcal{M}(S)$ recursively as follows

$$
U_{n+1}(A)=U_{n}(A)+R\left(Z_{n}, A\right), \quad A \in \mathcal{S},
$$

and

$$
\mathbb{P}\left(Z_{n} \in d s \mid U_{n}, U_{n-1}, \cdots, U_{0}\right)=\frac{U_{n}(d s)}{n+1} .
$$

Notice that, if $S$ is countable then $R$ can be presented as a stochastic matrix and then $R\left(Z_{n}, \cdot\right)$ is the $Z_{n}$-th row of the replacement matrix $R$. We will refer to the process $\left(U_{n}\right)_{n \geq 0}$ as the urn model with colors index by $S$, initial configuration $U_{0}$ and replacement kernel $R$. 
It is worth mentioning here that a little more general model may be obtained by taking $U_{0} \in \mathcal{M}(S)$ and not just in $\mathcal{P}(S)$. However, asymptotic results for $U_{n}$, when $U_{0} \in \mathcal{M}(S)$ can be easily derived from the special case $U_{0} \in \mathcal{P}(S)$.

Random configuration of the urn: Observe that since $R$ is a stochastic kernel and $U_{0} \in \mathcal{P}(S)$, we have

$$
U_{n}(S)=n+1
$$

for all $n \geq 0$. With slight abuse of terminology, we will call the random probability measure $\frac{U_{n}}{n+1}$, as the random configuration of the urn. In fact,

$$
\mathbb{P}\left(Z_{n} \in A \mid U_{n}, U_{n-1}, \cdots, U_{0}\right)=\frac{U_{n}(A)}{n+1}, A \in \mathcal{S} .
$$

In other words, the $n$-th random configuration of the urn is the conditional distribution of the $(n+1)$-th selected color, given $U_{0}, U_{1}, \ldots, U_{n}$.

Expected configuration of the urn: Taking expectation in equation (3), we get

$$
\mathbb{E}\left[U_{n}\right](S)=\mathbb{E}\left[U_{n}(S)\right]=n+1,
$$

for all $n \geq 0$. Thus $\frac{\mathbb{E}\left[U_{n}\right]}{n+1}$ is also a probability measure on $(S, \mathcal{S})$. In fact, it is the distribution of $Z_{n}$, the $(n+1)$-th selected color. This follows by taking expectation on both sides of equation (4),

$$
\mathbb{P}\left(Z_{n} \in A\right)=\frac{\mathbb{E}\left[U_{n}\right](A)}{n+1}, A \in \mathcal{S} .
$$

1.2. Notations. Most of the notations used in this paper are consistent with the literature on finite color urn models. However, we use few specific notations as well, which are given below.

(i) All vectors are written as row vectors unless otherwise stated. Column vectors are denoted by $x^{T}$, where $x$ is a row vector.

(ii) For any vector $x, x^{2}$ will denote a vector with the coordinates squared.

(iii) The standard Gaussian measure on $\mathbb{R}^{d}$ will be denoted by $\Phi_{d}$ with its density given by

$$
\phi_{d}(x):=\frac{1}{(2 \pi)^{d / 2}} \exp \left(-\frac{\|x\|^{2}}{2}\right), x \in \mathbb{R}^{d} .
$$

For $d=1$, we will simply write $\Phi$ for the standard Gaussian measure on $\mathbb{R}$ and $\phi$ for its density.

(iv) The symbol $\Rightarrow$ will denote convergence in distribution of random variables.

(v) The symbol $\stackrel{p}{\longrightarrow}$ will denote convergence in probability.

(vi) The symbol $\stackrel{w}{\longrightarrow}$ will denote the weak convergence of probability measures in $\mathcal{P}(S)$.

1.3. Outline of the Paper. The rest of the paper is organized as follows: Section 2 contains the two representation theorems, namely, Theorem 2.1] and Theorem 2.2, which are the most important contribution of this work. In Section 3, we derive asymptotic results for the random and expected configurations under fairly general conditions. In Section 4, we provide many interesting applications mainly in the context of infinite color urn schemes. 


\section{Main Results and Their Proofs}

In this section, we present the two main theorems of this paper. These theorems, which we call the Grand Representation Theorem (Theorem 2.1) and the Marginal Representation Theorem (Theorem 2.2 provide certain "couplings" of the urn model with the associated Markov chain through the observed sequence of colors. These results are fairly general and hold for any balance urn schemes with colors indexed by an arbitrary set $S$.

2.1. Grand Representation Theorem. The following theorem is a "representation" of the entire sequence of colors $\left(Z_{n}\right)_{n \geq 0}$ in terms of the associated Markov chain.

Theorem 2.1. Consider an urn model with colors indexed by a set $S$ endowed with a $\sigma$-algebra $\mathcal{S}$. Let $R$ be the replacement kernel and $U_{0}$ be the initial configuration. For $n \geq 0$, let $Z_{n}$ be the random color of the $(n+1)$-th draw. Further, for $n \geq-1$, let $\mathcal{T}_{n}$ be the random recursive tree on $(n+2)$ vertices labeled by $\{-1 ; 0,1, \cdots, n\}$ with -1 as the root. Let $\left(W_{n}\right)_{n \geq-1}$ be the branching Markov chain on $\mathcal{T}:=\cup_{n \geq-1} \mathcal{T}_{n}$ starting at the root -1 and at a position $\Delta \notin S$ with Markov transition kernel $\hat{R}$ on $(\hat{S}, \hat{\mathcal{S}})$, where $\hat{S}:=\{\Delta\} \cup S$ and $\hat{\mathcal{S}}:=\mathcal{S} \cup\{A \cup\{\Delta\} \mid A \in \mathcal{S}\}$, is given by

$$
\hat{R}(\hat{s}, A):=\left\{\begin{array}{lll}
U_{0}(A) & \text { if } & \hat{s}=\Delta ; \\
R(\hat{s}, A) & \text { if } \quad \hat{s} \neq \Delta,
\end{array}\right.
$$

for $\hat{s} \in\{\Delta\} \cup S$ and $A \in \mathcal{S}$. Then

$$
\left(Z_{n}\right)_{n \geq 0} \stackrel{d}{=}\left(W_{n}\right)_{n \geq 0} .
$$

Proof. Observe that from (1), we get for $n \geq 1$,

$$
\frac{U_{n}}{n+1}=\frac{U_{0}}{n+1}+\sum_{k=0}^{n-1} \frac{R\left(Z_{k}, \cdot\right)}{n+1} .
$$

Moreover, the conditional distribution of $Z_{n}$ given $Z_{0}, Z_{1}, \cdots, Z_{n-1}$ is given by the random configuration of the urn, namely, $\frac{U_{n}}{n+1}$. Now for $n \geq 0$, let $D_{n}$ be a discrete uniform random variable on the set of indices $\{-1 ; 0,1, \cdots, n-1\}$ such that the sequence $\left(D_{n}\right)_{n>0}$ are independent and for each $n \geq 0$ the random variable $D_{n}$ is independent of $\bar{Z}_{0}, Z_{1}, \cdots, Z_{n-1}$. Then

$$
\left(Z_{n} \mid Z_{n-1}, Z_{n-2}, \cdots, Z_{1}, Z_{0}\right) \stackrel{d}{=} R\left(Z_{D_{n}}, \cdot\right) .
$$

Further from definition we get

$$
\left(W_{n} \mid W_{n-1}, W_{n-2}, \cdots, W_{1}, W_{0}\right) \stackrel{d}{=} R\left(W_{D_{n}}, \cdot\right) .
$$

This completes the proof.

2.2. Marginal Representation Theorem. Our next result is a "representation" of the marginal distribution for the randomly chosen color $Z_{n}$ in terms of the marginal distribution of the corresponding Markov chain sampled at random but independent times. As we will see from the proof it is an immediate corollary of Theorem 2.1. 
Theorem 2.2. Consider an urn model with colors indexed by a set $S$ endowed with a $\sigma$-algebra $\mathcal{S}$. Let $R$ be the replacement kernel and $U_{0}$ be the initial configuration. For $n \geq 0$, let $Z_{n}$ be the random color of the $(n+1)$-th draw. Let $\left(X_{n}\right)_{n>0}$ be the associated Markov chain on $S$ with transition kernel $R$ and initial distribution $U_{0}$. Then there exists an increasing non-negative sequence of stopping times $\left(\tau_{n}\right)_{n \geq 0}$ with $\tau_{0}=0$, which are independent of the Markov chain $\left(X_{n}\right)_{n \geq 0}$, such that,

$$
Z_{n} \stackrel{d}{=} X_{\tau_{n}}
$$

for any $n \geq 0$. Moreover, as $n \rightarrow \infty$,

$$
\frac{\tau_{n}}{\log n} \longrightarrow 1 \text { a.s. }
$$

and

$$
\frac{\tau_{n}-\log n}{\sqrt{\log n}} \stackrel{d}{\longrightarrow} N(0,1) .
$$

Remark: Recall that the probability mass function of $Z_{n}$ is $\frac{1}{n+1} \mathbf{E}\left[U_{n}\right]$, which implies that (12) will be useful in deriving results for the expected configuration. A version of this result was obtained in Proposition 7 in [5], which was restricted to the case when $\left(X_{n}\right)_{n \geq 0}$ is a bounded increment random walk. Here however, the result is for any general Markov chain $\left(X_{n}\right)_{n \geq 0}$.

Remark: It is worthwhile to note here that the following may not be necessarily true

$$
\left(Z_{n}\right)_{n \geq 0} \stackrel{d}{=}\left(X_{\tau_{n}}\right)_{n \geq 0}
$$

This is because $\left(Z_{n}\right)_{n \geq 0}$ is not necessarily Markov, but $\left(X_{\tau_{n}}\right)_{n \geq 0}$ is Markovian. In fact, the law of the process $\left(Z_{n}\right)_{n \geq 0}$ is more complicated as presented in Theorem 2.1 .

Proof. Let $\left(1+\tau_{n}\right)$ be length of the unique path from the vertex $n$ to the root -1 in the random recursive tree $\mathcal{T}_{n}$ with $n+1$ vertices, as defined in the Theorem 2.1. Then from definition it follows that

$$
W_{n} \stackrel{d}{=} X_{\tau_{n}},
$$

and thus 12 follows from Theorem 2.1

Now, for $0 \leq j \leq n-1$, let $I_{j}$ be the indicator that the vertex $j$ lies on the path from the root -1 to the vertex $n$. Then by construction $\left(I_{j}\right)_{0 \leq j \leq n-1}$ are independent Bernoulli variables with $\mathbf{E}\left[I_{j}\right]=\frac{1}{j+2}, 0 \leq j \leq n-1$. Also,

$$
\tau_{n}=\sum_{j=0}^{n-1} I_{j}
$$

Notice that $\operatorname{var}\left(I_{j}\right)=\frac{1}{j+2}\left(1-\frac{1}{j+2}\right), 0 \leq j \leq n-1$, thus

$$
\mathbf{E}\left[\tau_{n}\right]=\sum_{j=0}^{n-1} \frac{1}{j+2} \sim \log n \text { and } \operatorname{var}\left(\tau_{n}\right)=\sum_{j=0}^{n-1} \frac{1}{j+2}\left(1-\frac{1}{j+2}\right) \sim \log n,
$$


as $n \rightarrow \infty$. So by Kronecker's Lemma (see (8.5) on page 63 of [17]) it follows that

$$
\frac{\tau_{n}}{\log n} \longrightarrow 1 \text { a.s. }
$$

as $n \rightarrow \infty$, proving (13). Further, (14) follows by an easy application of the Lyapunov Central Limit Theorem (see Theorem 27.3 on page 362 of [6]).

\section{Weak Asymptotic of the URn Configuration}

In this section we state and prove some very general results for the asymptotic of the random and expected configurations of our general urn scheme $\left(U_{n}\right)_{n \geq 0}$, defined in Section 1.1. These results will be proved using the two representations theorems given in Section 2. We start by establishing an asymptotic result for the branching Markov chain $\left(W_{n}\right)_{n>-1}$ as defined in Theorem 2.1. For this and the later sections, we assume that $\mathcal{P}(S)$ is endowed with the topology of weak convergence and any limit statement in $\mathcal{P}(S)$ is with respect to the topology of weak convergence.

3.1. Asymptotic of Branching Markov Chain on Random Recursive Tree. Let us first recall that $\left(W_{n}\right)_{n>-1}$ is defined as the branching Markov chain on the (infinite) random recursive tree $\mathcal{T}:=\cup_{n \geq-1} \mathcal{T}_{n}$, starting at the root -1 and at a position $\Delta \notin S$ with Markov transition kernel $\hat{R}$ on $(\hat{S}, \hat{\mathcal{S}})$ given in (7). Define $\mathcal{G}_{n}:=\sigma\left(W_{0}, W_{1}, \cdots, W_{n-1}\right), n \geq 0$. Let $Q_{n}$ be a version of the regular conditional distribution of $W_{n}$ given $\mathcal{G}_{n}$. Note that $Q_{n}$ exists and is almost surely unique and proper, as $S$ is a Polish space and $\mathcal{S}$ is the corresponding Borel $\sigma$-algebra. Further, let $q_{n}$ be a version of the regular conditional distribution of $W_{n}$ given $\mathcal{G}_{n}$ and $\mathcal{T}_{n}$. Once again $q_{n}$ exists and is almost surely unique and proper. It is worth noting here that for any $A \in \mathcal{S}, \mathbf{E}\left[q_{n}(A) \mid \mathcal{G}_{n}\right]=Q_{n}(A)$.

Further, recall $1+\tau_{n}$ is defined to be the length of the unique path from the vertex $n$ to the root -1 in the random recursive tree $\mathcal{T}$.

Also recall that $\left(X_{n}\right)_{n \geq 0}$ denotes a Markov chain with state space $S$, transition kernel $R$ and starting distribution $U_{0}$.

We now make the following assumption:

(A) There exists a (non-random) probability $\Lambda$ on $\left(\mathbb{R}^{d}, \mathcal{B}_{\mathbb{R}^{d}}\right)$ and a vector $\mathbf{v} \in$ $\mathbb{R}^{d}$, and two functions $a: \mathbb{R}_{+} \rightarrow \mathbb{R}$ and $b: \mathbb{R}_{+} \rightarrow \mathbb{R}_{+}$, such that, for any starting distribution $U_{0}$,

$$
\frac{X_{n}-a(n) \mathbf{v}}{b(n)} \Rightarrow \Lambda \text {. }
$$

Theorem 3.1. Suppose assumption $(\boldsymbol{A})$ holds. Let $q_{n}^{c s}$ be the conditional distribution of $\frac{W_{n}-a\left(\tau_{n}\right) \mathbf{v}}{b\left(\tau_{n}\right)}$ given $\mathcal{G}_{n}$ and $\mathcal{T}_{n}$, that is, a scaled and centered version of $q_{n}$ with centering $a\left(\tau_{n}\right) \mathbf{v}$ and scaling $b\left(\tau_{n}\right)$. Then as $n \rightarrow \infty$,

$$
q_{n}^{c s} \stackrel{p}{\longrightarrow} \Lambda \text { in } \mathcal{P}\left(\mathbb{R}^{d}\right) \text {. }
$$

Moreover, let $Q_{n}^{c s}$ is the conditional distribution of $\frac{W_{n}-a(\log n) \mathbf{v}}{b(\log n)}$ given $\mathcal{G}_{n}$, that is, a scaled and centered version of $Q_{n}$ with centering by $a(\log n) \mathbf{v}$ and scaling by $b(\log n)$, then 
(a) If $a \equiv 0$ and $b \equiv 1$, then

$$
Q_{n}^{c s}=Q_{n} \stackrel{p}{\longrightarrow} \Lambda \text { in } \mathcal{P}(S) .
$$

(b) Suppose $a=0$ and $b$ is regularly varying function, then

$$
Q_{n}^{c s} \stackrel{p}{\longrightarrow} \Lambda \text { in } \mathcal{P}\left(\mathbb{R}^{d}\right) .
$$

(c) Suppose $a$ is differentiable and $\lim _{x \rightarrow \infty} a^{\prime}(x)=\tilde{a}<\infty$. Also assume $b$ is regularly varying and $\lim _{x \rightarrow \infty} \frac{\sqrt{x}}{b(x)}=\tilde{b}<\infty$ then

$$
Q_{n}^{c s} \stackrel{p}{\longrightarrow} \Xi \text { in } \mathcal{P}\left(\mathbb{R}^{d}\right),
$$

where $\Xi$ is $\Lambda$ if $\tilde{a}=0$ or $\tilde{b}=0$, otherwise, it is given by the convolution of $\Lambda$ and $\operatorname{Normal}\left(0, \tilde{a}^{2} \tilde{b}^{2}\right) \mathbf{v}$.

Proof. Let $\rho$ be a metric on $\mathcal{P}(S)$ which metrize the weak convergence topology on it. Denote by $L_{n}$ the distribution of $\frac{X_{n}-a(n)}{b(n)}$. Under assumption (A), we have

$$
\rho\left(L_{n}, \Lambda\right) \longrightarrow 0
$$

as $n \rightarrow \infty$.

Now, fix $\epsilon>0$ and find $H>0$ so large that $\rho\left(L_{h}, \Lambda\right)<\epsilon$, for any $h>H$. Find $N \geq 1$ so large that $\frac{(\log (n+2))^{H}}{n+2}<\epsilon$ for all $n \geq N$.

Let $S_{n}^{H}$ be the set of vertices of the random recursive tree $\mathcal{T}_{n}$ up to depth $H$. Then $\mathbf{E}\left[\left|S_{n}^{H}\right|\right]=(\log (n+2))^{H}+o(1)$.

Recall that $D_{n}$ denotes the vertex at which $n$-th vertex joins in the random recursive tree $\mathcal{T}_{n}$. Then by construction

$$
\mathbf{P}\left(\rho\left(q_{n}^{\mathrm{cs}}, \Lambda\right)>\epsilon\right) \leq \mathbf{P}\left(D_{n} \in S_{n}^{H}\right)=\frac{\mathbf{E}\left[S_{n}^{H}\right]}{n+2}<\epsilon .
$$

This completes the proof of the first part.

For proof of part (a), it is enough to observe that under the assumptions of $a=0$ and $b=1, Q_{n}^{\mathrm{cs}}=\mathbf{E}\left[q_{n}^{\mathrm{cs}} \mid \mathcal{G}_{n}\right]$ almost surely.

For part (b), we first observe that if $b$ is regularly varying, then by Karamata's Characterization Theorem 21 and equation 19 we can show that

$$
\frac{b(\log n)}{b\left(\tau_{n}\right)} \stackrel{p}{\longrightarrow} 1 \text {. }
$$

Further by the delta-method [17, under assumptions in part (c),

$$
\frac{a\left(\tau_{n}\right)-a(\log n)}{\sqrt{\log n}} \Rightarrow \begin{cases}\operatorname{Normal}(0, \tilde{a}) & \text { if } \tilde{a} \neq 0 \\ \delta_{0} & \text { otherwise }\end{cases}
$$

Finally, we note that

$$
\frac{W_{n}-a\left(\tau_{n}\right) \mathbf{v}}{b\left(\tau_{n}\right)}=\frac{b(\log n)}{b\left(\tau_{n}\right)}\left(\frac{W_{n}-a(\log n) \mathbf{v}}{b(\log n)}+\frac{\sqrt{\log n}}{b(\log n)} \frac{a\left(\tau_{n}\right)-a(\log n)}{\sqrt{\log n}}\right) .
$$


3.2. Asymptotic of the Random Configuration of the Urn. Define $\mathcal{F}_{n}:=$ $\sigma\left(Z_{0}, Z_{1}, \cdots, Z_{n}\right), n \geq 0$. Let $P_{n}$ be a version of the regular conditional distribution of $Z_{n}$ given $\mathcal{F}_{n}$. Note by construction $P_{n}=\frac{U_{n}}{n+1}$ almost surely. The following result is an immediate corollary of the Theorem 2.1 and Theorem 3.1 .

Theorem 3.2. Suppose assumption $(\boldsymbol{A})$ holds. Let $P_{n}^{c s}$ is the conditional distribution of $\frac{Z_{n}-a(\log n) \mathbf{v}}{b(\log n)}$ given $\mathcal{F}_{n}$, that is, a scaled and centered version of $P_{n}$ with centering by $a(\log n) \mathbf{v}$ and scaling by $b(\log n)$, then

(a) If $a=0$ and $b=1$, then

$$
P_{n}^{c s}=P_{n} \stackrel{p}{\longrightarrow} \Lambda \text { in } \mathcal{P}(S) .
$$

(b) If conditions of the Part (b) of the Theorem 3.1 hold, then

$$
P_{n}^{c s} \stackrel{p}{\longrightarrow} \Lambda \text { in } \mathcal{P}\left(\mathbb{R}^{d}\right) \text {. }
$$

(c) If conditions of the Part (c) of the Theorem 3.1 hold, then

$$
P_{n}^{c s} \stackrel{p}{\longrightarrow} \Xi \text { in } \mathcal{P}\left(\mathbb{R}^{d}\right),
$$

where $\Xi$ is $\Lambda$ if $\tilde{a}=0$ or $\tilde{b}=0$, otherwise, it is given by the convolution of $\Lambda$ and $\operatorname{Normal}\left(0, \tilde{a}^{2} \tilde{b}^{2}\right) \mathbf{v}$.

3.3. Asymptotic of the Expected Configuration of the Urn. Recall that $\mathbf{E}\left[P_{n}\right]=\frac{\mathbf{E}\left[U_{n}\right]}{n+1}$ is the marginal distribution of $Z_{n}$. The following result is an immediate corollary of the Theorem 3.2 .

Theorem 3.3. Suppose assumption (A) holds, then

(a) If $a=0$ and $b=1$, then

$$
Z_{n} \Rightarrow \Lambda \text {. }
$$

(b) If conditions of the Part (b) of the Theorem 3.1 hold, then

$$
\frac{Z_{n}-a(\log n) \mathbf{v}}{b(\log n)} \Rightarrow \Lambda
$$

(c) If conditions of the Part (c) of the Theorem 3.1 hold, then

$$
\frac{Z_{n}-a(\log n) \mathbf{v}}{b(\log n)} \Rightarrow \Xi
$$

where $\Xi$ is $\Lambda$ if $\tilde{a}=0$ or $\tilde{b}=0$, otherwise, it is given by the convolution of $\Lambda$ and Normal $\left(0, \tilde{a}^{2} \tilde{b}^{2}\right) \mathbf{v}$.

Proof. The result follows from the Theorem 3.2 by taking expectation and noting the fact that centering and scaling are non-random in all cases.

\section{Applications}

In this section we discuss several applications of the representation theorems (Theorem 2.1 and Theorem 2.2. Essentially all the results stated here are proved using the two general asymptotic results, namely, Theorem 3.2 and Theorem 3.3 . given in the previous section. 
4.1. $S$ is Countable and $R$ is Ergodic. Suppose the indexing set of colors $S$ is either finite or countably infinite and we endow $S$ with the sigma-algebra $\mathcal{S}$, which is the power set $\wp(S)$. In this case, we can view the Markov transition kernel $R$ as a matrix and it is then called the replacement matrix. For $S$ finite, it is the classical case. If we assume that $R$ is ergodic, that is, assumption (A) holds with $a=0$ and $b=1$, then from Theorem 3.2 (a) we get the following result.

Theorem 4.1. Suppose $S$ is countable, $\mathcal{S}=\wp(S), R$ is ergodic with stationary distribution $\pi$ on $S$. Then as $n \rightarrow \infty$,

$$
\frac{U_{n}}{n+1} \stackrel{p}{\longrightarrow} \pi \text { in } \mathcal{P}(S) \text {. }
$$

In particular,

$$
\frac{\mathbf{E}\left[U_{n}\right]}{n+1} \stackrel{w}{\longrightarrow} \pi \text { in } \mathcal{P}(S),
$$

as $n \rightarrow \infty$.

If $S$ is finite then using either matrix algebra techniques or multi-type branching process techniques, it is known [22, 23] that stronger result holds. In fact, under even weaker assumption of only irreducibility of the chain, the convergence in probability in (36) can be replaced by almost sure convergence. We believe that in general for $S$ countable, under ergodicity assumption almost sure convergence should hold. We here note that as soon as $S$ is infinite, the classical techniques such as matrix algebra methods using Perron-Frobenius theory of matrices with positive entries [31] and Jordan Decomposition of finite dimensional matrices [15, or martingale approach using embedding to multi-type branching processes, which have been extensively used in classical urn model literature [1, 3, 8, 16, 22, 24; fails to derive any result. We are hopeful that our novel and fairly probabilistic approach, namely, the Grand and Marginal representation Theorems should yield the classical result. Unfortunately, we have been unable to derive it so far.

4.2. $S$ is Countable and $R$ is Block Diagonal. Similar to the previous section, suppose the indexing set of colors $S$ is either finite or countably infinite and we endow $S$ with the sigma-algebra $\mathcal{S}$, which is the power set $\wp(S)$. As in the previous case, we view the Markov transition kernel $R$ as a matrix. Suppose the replacement matrix $R$, can be decomposed in the following manner. Let the indexing set of colors be partitioned as $S=\underset{i \in \mathcal{I}}{\cup} C_{i}$, where $\mathcal{I}$ is a countable set, and $C_{i}$ is countable for all $i \in \mathcal{I}$. We endow $\mathcal{I}$ with its power set as a $\sigma$-algebra on it and each $C_{i}$ is also endowed with its power set as the $\sigma$-algebra on it.

Now let, $\phi: S \rightarrow \mathcal{I}$ be the "projection" map, which maps $s \mapsto i$, where $i$ is the unique element of $\mathcal{I}$, such that, $s \in C_{i}$.

Now, suppose for every $i \in \mathcal{I}$ and $s \in C_{i}$, the kernel $R(s, \cdot)$ is a probability measure supported only on $C_{i}$, that is,

$$
R\left(s, C_{i}\right)= \begin{cases}1 & \text { if } s \in C_{i} \\ 0 & \text { otherwise }\end{cases}
$$

As each $C_{i}$ is countable, $R$ on $C_{i}$ can be realized as a (possibly infinite) matrix $R_{i i}$ indexed by the colors in $C_{i}$. 
Note that if $S$ is finite then $R$ is essentially a reducible matrix with diagonal blocks, which can be presented as

$$
R=\left(\begin{array}{ccccc}
R_{11} & 0 & 0 & \cdots & 0 \\
0 & R_{22} & 0 & \cdots & 0 \\
0 & 0 & R_{33} & \cdots & 0 \\
\vdots & \vdots & \vdots & \ddots & \vdots \\
0 & 0 & 0 & \cdots & R_{k k}
\end{array}\right)
$$

We further assume that the for all $i \in \mathcal{I}$, the kernel/replacement matrix, $R_{i i}$ restricted to its "block" $C_{i}$, is ergodic with stationary distribution, $\pi_{i}$.

Theorem 4.2. Consider an urn model with colors indexed by a set $S$ and replacement kernel $R$ as in (38). Then for every initial configuration $U_{0}$, as $n \rightarrow \infty$,

$$
\frac{U_{n}}{n+1} \stackrel{p}{\longrightarrow} \Pi \text { in } \mathcal{P}(S)
$$

where $\Pi$ is a random probability measure on $(S, \mathcal{S})$ given by

$$
\Pi(A)=\sum_{i \in \mathcal{I}} \pi_{i}\left(A \cap C_{i}\right) \nu_{i}, \quad A \in \mathcal{S},
$$

and $\nu$ has Ferguson Distribution on the countable set $\mathcal{I}$ with parameter $U_{0} \circ \phi^{-1}$.

Proof. Let us denote by $c_{i}:=\sum_{v \in C_{i}} U_{0, v}$, and $T_{n, i}:=\sum_{v \in C_{i}} U_{n, v}$ for each $i \geq 1$. It is easy to check that for each $i \geq 1$, the sequence $\left(\frac{T_{n, i}}{n+1}\right)_{n>0}$ is non-negative a.s. convergent martingale, see 22 for details. Consider each $C_{i}$ to be a super color for $i \geq 1$. Then $T_{n}:=\left(T_{n, i}\right)_{i \geq 1}$ corresponds to the configuration of a classical Pólya urn model, with initial configuration $T_{0}=\left(c_{i}\right)_{i \geq 1}$. It is worthwhile to note here that $\frac{T_{n}}{n+1}$ is a probability measure on $\mathcal{I}$. Therefore, from [7], as $n \rightarrow \infty$

$$
\frac{T_{n}}{n+1} \longrightarrow \nu \text { a.s. }
$$

where $\nu$ is a random measure on $\mathcal{I}$ having Ferguson Distribution with parameter $U_{0} \circ \phi^{-1}$.

Define $N_{n, i}:=\sum_{k=1}^{n} \mathbf{1}_{Z_{k} \in C_{i}}$. Then it is obvious that $T_{n, i}=c_{i}+N_{n, i}$. Writing $U_{n}=\left(U_{n, C_{i}}\right)_{i \geq 1}$, we observe that for each $i \geq 1,\left(U_{N_{n, i}, C_{i}}\right)$ is the configuration of an urn with initial configuration $\frac{U_{0, C_{i}}}{c_{i}}$ and replacement matrix $R_{i i}$. Since from (41) we know that $N_{n, i} \longrightarrow \infty$ a.s. as $n \rightarrow \infty$, it follows from Theorem 4.1 that as $n \rightarrow \infty$,

$$
\frac{U_{N_{n, i}, C_{i}}}{T_{n, i}} \stackrel{P}{\longrightarrow} \pi_{i} \text { in } \mathcal{P}(S),
$$

This implies that

$$
\frac{U_{n, C_{i}}}{n+1}=\frac{U_{N_{n, i}, C_{i}}}{n+1}=\frac{U_{N_{n, i}, C_{i}}}{T_{n, i}} \frac{T_{n, i}}{n+1} \stackrel{P}{\longrightarrow} \nu\left(C_{i}\right) \pi_{i}
$$

where $\nu$ as in (41). This completes the proof. 
4.3. Urn Models Associated with Random Walks on $\mathbb{Z}^{d}$. It this section we consider urn models associated with random walks on $\mathbb{Z}^{d}$. These models were first introduced in [5], where only bounded increment walks were considered. In general, here we take $S=\mathbb{Z}^{d}$ for some $d \geq 1$ and $\mathcal{S}$ will be taken as the power set of $\mathbb{Z}^{d}$. The kernel $R$ can be viewed as an infinite dimensional matrix index by the set of colors $\mathbb{Z}^{d}$, given by

$$
R(u, v)=\mathbf{p}(v-u), u, v \in \mathbb{Z}^{d},
$$

where $\mathbf{p}$ is the distribution on $\mathbb{Z}^{d}$ of the independent increments of the walk.

4.3.1. Finite Variance Walks. Suppose $\mathbf{p}$ has finite second moment, leading to a random walk with finite variance. Following theorem is a generalization of the results derived in [5].

Theorem 4.3. Consider an infinite color urn model with colors indexed by $S=\mathbb{Z}^{d}$, and kernel $R$ as given above. Suppose the starting configuration is $U_{0}$. Then there exist $\mu \in \mathbb{R}^{d}$ and a positive definite matrix $\Sigma_{d \times d}$, such that, if we define,

$$
P_{n}^{c s}(A):=\frac{U_{n}}{n+1}\left(\sqrt{\log n} A \Sigma^{1 / 2}+\mu \log n\right), \quad A \in \mathcal{B}_{\mathbb{R}^{d}},
$$

where

$$
x A \Sigma^{1 / 2}:=\left\{x y \Sigma^{1 / 2}: y \in A\right\}
$$

then, as $n \rightarrow \infty$,

$$
P_{n}^{c s} \stackrel{p}{\longrightarrow} \Phi_{d} \text { in } \mathcal{P}\left(\mathbb{R}^{d}\right)
$$

In particular,

$$
\frac{Z_{n}-\mu \log n}{\sqrt{\log n}} \Rightarrow \operatorname{Normal}_{d}(0, \Sigma),
$$

as $n \rightarrow \infty$

Proof. Let $X_{n}$ be the position of the random walk starting with $X_{0} \sim U_{0}$ and independent increments with distribution given by p. From classical Central Limit Theorem [17], we get that

$$
\frac{X_{n}-n \mu}{\sqrt{n}} \Rightarrow \operatorname{Normal}_{d}\left(\mathbf{0}, \Sigma-\mu \mu^{T}\right),
$$

where $\mu$ is the mean of the increment distribution and $\Sigma$ is the second moment. Thus assumption (A) holds, with $\mathbf{v}=\mu, a(n)=n, b(n)=\sqrt{n}$ and $\Lambda=$ Normal $_{d}\left(\mathbf{0}, \Sigma-\mu \mu^{T}\right)$.

We observe that the assumptions in Part (c) of Theorem 3.1 holds with $\tilde{a}=1$ and $\tilde{b}=1$. This completes the proof of $\sqrt[45]{ }$, by observing that $\Xi=\operatorname{Normal}_{d}(0, \Sigma)$.

Finally, 46 follows from Theorem 3.3.(c).

4.3.2. Symmetric $\alpha$-Stable Walks. As an example of an infinite variance case, which could not have been done by the techniques derived in [5], we consider the case $\mathbf{p}$ is a symmetric $\alpha$-stable distribution on $\mathbb{Z}$, for sake of completeness we provide here the definition.

Definition 4.4. A distribution $\mathbf{p}$ is said to have a symmetric $\alpha$-stable distribution, denoted by $S \alpha S$, with $0<\alpha \leq 2$, if for any $t \in \mathbb{R}$,

$$
\mathbf{E}\left[e^{i t Y}\right]=\exp \left(-\sigma^{\alpha}|t|^{\alpha}\right)
$$

for some $\sigma>0$, where $Y \sim \mathbf{p}$. 
We further assume that $\mathbf{p}$ has the following properties. If $Y \sim \mathbf{p}$, then

(i) There exists $0<\alpha \leq 2$, and a slowly varying function $L(\cdot)$, such that

$$
n^{\alpha} \mathbf{P}(|Y|>n)=L(n), \text { for all } n \in \mathbb{N} ; \text { and }
$$

(ii)

$$
\lim _{n \rightarrow \infty} \frac{\mathbf{P}(Y>n)}{\mathbf{P}(|Y|>n)}=\frac{1}{2}
$$

Theorem 4.5. Consider an infinite color urn model with colors indexed by $S=\mathbb{Z}^{d}$, and kernel $R$ defined by (44), where $\mathbf{p}$ is as defined above. Suppose the starting configuration is $U_{0}$. Then there exist sequences $\left(c_{n}\right)_{n \geq 1}$ and $\left(s_{n}\right)_{n \geq 1}$, and a $S \alpha S$ distribution $\Lambda$, where $\alpha$ is as in (49), such that, if we define, $P_{n}^{c s}$ as the conditional distribution of $\frac{Z_{n}-c_{n}}{s_{n}}$ given $\mathcal{F}_{n}$, then, as $n \rightarrow \infty$,

$$
P_{n}^{c s} \stackrel{p}{\longrightarrow} \Lambda \text { in } \mathcal{P}\left(\mathbb{R}^{d}\right) .
$$

In particular,

$$
\frac{Z_{n}-c_{n}}{s_{n}} \Rightarrow \Lambda
$$

as $n \rightarrow \infty$.

Moreover, we may choose $s_{n}=(\log n)^{\frac{1}{\alpha}} h(\log n), n \geq 1$, where $h(\cdot)$ is a suitable slowly varying function, and $c_{n}=0, \forall n \geq 1$, when $0<\alpha \leq 1$; and $c_{n}=\mathbf{E}[Y] \log n, n \geq 1$, with $Y \sim \mathbf{p}$, for $1<\alpha \leq 2$.

Proof. Let $X_{n}$ be the position of the random walk starting with $X_{0} \sim U_{0}$ and independent increments with distribution given by $\mathbf{p}$. From [21], we get that

$$
\frac{X_{n}-a(n) \mathbf{v}}{b(n)} \Rightarrow \Lambda
$$

where $\Lambda$ is a $S \alpha S$-distribution with $\alpha$ is as in (49). Moreover, we may choose,

$$
a(x)= \begin{cases}0 & \text { if } 0 \leq \alpha \leq 1 \\ x & \text { otherwise }\end{cases}
$$

for $x \geq 0$, and

$$
\mathbf{v}= \begin{cases}0 & \text { if } 0 \leq \alpha \leq 1 \\ \mathbf{E}[Y] & \text { otherwise }\end{cases}
$$

where $Y \sim \mathbf{p}$, and

$$
b(x)=x^{\frac{1}{\alpha}} h(x), x \in \mathbb{R}_{+},
$$

for some slowly varying function $h: \mathbb{R}_{+} \rightarrow \mathbb{R}_{+}$. This completes the proof by using Theorem 3.2(b) \& (c) and Theorem 3.3(b) \& (c), by observing that in this case $\tilde{b}=0$. 


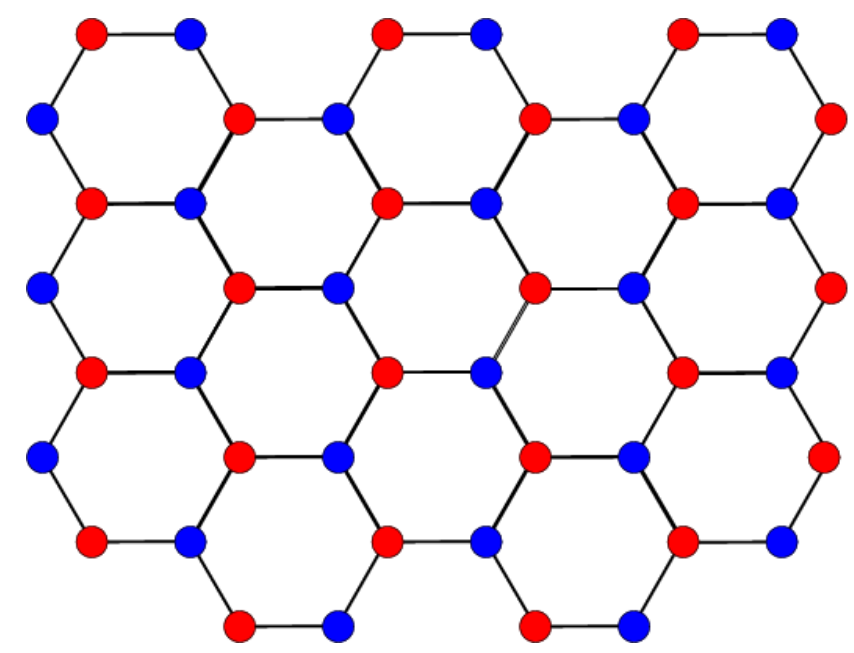

Figure 1. Hexagonal Lattice

4.4. Urn Models Associated with Periodic Random Walk on $\mathbb{R}^{d}$. Let $\mathbb{H}=$ $(V, E)$ be the hexagonal lattice in $\mathbb{R}^{2}$ [see Figure 1 . The vertex set can easily be partitioned into two non-empty parts, $V=V_{1} \cup V_{2}$, where $V_{1}$ and $V_{2}$ are disjoint, and the random walk $\mathbb{H}$ is then a periodic chain. If the replacement kernel be denoted by $R$, then corresponding urn scheme with colors indexed by $\mathbb{H}$, is not covered by the earlier stated Theorem 4.3 . For studying such cases, we consider the following slightly more general type of random walk on $\mathbb{R}^{d}$.

Let $\left\{Y_{j}(i), 1 \leq i \leq k, j \geq 1\right\}$ be a collection of independent random $d$-dimensional vectors, such that, for each fixed $i \in\{1,2, \ldots k\},\left(Y_{j}(i)\right)_{j \geq 1}$ are i.i.d. We further assume that for each fixed $1 \leq i \leq k$, there exists a finite non-empty set $B_{i} \subset \mathbb{R}^{d}$, such that, $\mathbf{P}\left(Y_{1}(i) \in B_{i}\right)=1$, and $B_{i} \cap B_{j}=\emptyset$, for any $1 \leq i, j \leq k$. That is, for each $i \in\{1,2, \ldots, k\}$, we assume that the law of $Y_{1}(i)$ is bounded. For $1 \leq i \leq k$, we shall write

$$
\begin{aligned}
\mu(i) & :=\mathbf{E}\left[Y_{1}(i)\right], \\
\bar{\mu} & :=\frac{1}{k} \sum_{i=1}^{k} \mu(i), \\
\Sigma(i) & :=\mathbf{E}\left[Y_{1}^{T}(i) Y_{1}(i)\right] .
\end{aligned}
$$

We further assume that $\Sigma(i)$ is positive definite, for each $1 \leq i \leq k$. Let us denote by $\Sigma^{1 / 2}(i)$ the unique positive definite square root of $\Sigma(i)$. Note that, then $\bar{\Sigma}=$ $\frac{1}{k} \sum_{i=1}^{k} \Sigma(i)$ is also positive definite. We denote by $\bar{\Sigma}^{1 / 2}$, the unique positive definite square root of $\bar{\Sigma}$.

For $n=m k+r$, where $m \in \mathbb{N} \cup\{0\}$, and $0 \leq r<k$, let

$$
X_{n}=X_{m k}+Y_{m+1}(1)+Y_{m+1}(2)+\ldots+Y_{m+1}(r+1),
$$

be the $k$-periodic random walk with increments $\left\{Y_{j}(i), 1 \leq i \leq k, j \geq 1\right\}$.

In the remainder of this subsection, we will consider an urn model $\left(U_{n}\right)_{n \geq 0}$, with colors indexed by $S=\mathbb{R}^{d}$, starting at some distribution $U_{0}$ on $\mathbb{R}^{d}$ and with a replacement kernel $R$ associated with a periodic random walk with periodic increments as given above. 
Theorem 4.6. Consider an infinite color urn model with colors indexed by $S=\mathbb{R}^{d}$, and kernel $R$ as given above. Suppose the starting configuration is $U_{0}$. If we define,

$$
P_{n}^{c s}(A):=\frac{U_{n}}{n+1}\left(\sqrt{\log n} A \bar{\Sigma}^{1 / 2}+\bar{\mu} \log n\right), \quad A \in \mathcal{B}_{\mathbb{R}^{d}},
$$

where

$$
x A \Sigma^{1 / 2}:=\left\{x y \Sigma^{1 / 2}: y \in A\right\},
$$

then, as $n \rightarrow \infty$,

$$
P_{n}^{c s} \stackrel{p}{\longrightarrow} \Phi_{d} \text { in } \mathcal{P}\left(\mathbb{R}^{d}\right)
$$

In particular,

$$
\frac{Z_{n}-\bar{\mu} \log n}{\sqrt{\log n}} \Rightarrow \operatorname{Normal}_{d}(0, \bar{\Sigma})
$$

as $n \rightarrow \infty$

Proof. We first note that by Theorem 3.2(c) and Theorem 3.3 (c), it is enough to show that

$$
\frac{X_{n}-n \bar{\mu}}{\sqrt{n}} \Rightarrow N_{d}(0, \bar{D})
$$

where $\bar{D}=\frac{1}{k} \sum_{i=1}^{k} \operatorname{var}\left(Y_{1}(i)\right)$. This follows from standard application of i.i.d. Central Limit Theorem [17.

As an application of the Theorem 4.6, we now consider our starting example of the random walk on hexagonal lattice. Let $\mathbb{H}=(V, E)$ be the hexagonal lattice in $\mathbb{R}^{2}$ [see Figure 1]. The vertex set $V=V_{1} \cup V_{2}$, where $V_{1}$ and $V_{2}$ are disjoint. $V_{1}$ and $V_{2}$ are defined as follows:

$$
V_{1,1}:=\left\{1, \omega, \omega^{2}\right\} \text {, where } \omega \text { is a complex cube root of unity, }
$$

and

For any $n \geq 2$,

$$
V_{2,1}:=\left\{v+1, v+\omega, v+\omega^{2}: v \in V_{1,1}\right\} .
$$

and

$$
V_{1, n}:=\left\{v-1, v-\omega, v-\omega^{2}: v \in V_{2, n-1}\right\},
$$

$$
V_{2, n}=\left\{v+1, v+\omega, v+\omega^{2}: v \in V_{1, n}\right\} .
$$

Finally, $V_{1}=\cup_{j \geq 1} V_{1, j}$ and $V_{2}=\cup_{j \geq 1} V_{2, j}$. For any pair of vertices $v, w \in V$, we draw an edge between them, if and only if, either of the following two cases occur:

(i) $v \in V_{1}$ and $w \in V_{2}$ and $w=v+u$ for some $u \in\left\{1, \omega, \omega^{2}\right\}$, or

(ii) $v \in V_{2}$ and $w \in V_{1}$ and $w=v+u$ for some $u \in\left\{-1,-\omega,-\omega^{2}\right\}$.

To define the random walk on $\mathbb{H}$, let us consider $\left\{Y_{j}(i): i=1,2, j \geq 1\right\}$ to be a sequence of independent random vectors such that $\left(Y_{j}(i)\right)_{j \geq 1}$ are i.i.d for every fixed $i=1,2$. Let $Y_{1}(1) \sim \operatorname{Unif}\left\{1, \omega, \omega^{2}\right\}$, and $Y_{1}(2) \sim \operatorname{Unif}\left\{-1,-\omega,-\omega^{2}\right\}$. One can now define a random walk on $\mathbb{H}$, with the increments $\left\{Y_{j}(i): i=1,2, j \geq 1\right\}$. Needless to say, this random walk has period 2.

Corollary 4.7. Consider an infinite color urn model with colors indexed by $S=\mathbb{H}$, and kernel $R$ as given above. Suppose the starting configuration is $U_{0}$. If we define,

$$
P_{n}^{c s}(A):=\frac{U_{n}}{n+1}(2 \sqrt{\log n} A), \quad A \in \mathcal{B}_{\mathbb{R}^{d}}
$$

then, as $n \rightarrow \infty$,

$$
P_{n}^{c s} \stackrel{p}{\longrightarrow} \Phi_{2} \text { in } \mathcal{P}\left(\mathbb{R}^{2}\right)
$$


In particular,

as $n \rightarrow \infty$

$$
\frac{2 Z_{n}}{\sqrt{\log n}} \Rightarrow \operatorname{Normal}_{2}\left(0, \mathbb{I}_{2}\right)
$$

Proof. First of all we note that, it is enough to show that $\bar{\Sigma}=\frac{1}{2} \mathbb{I}_{2}$.

Now, since $1+\omega+\omega^{2}=0$, so for the random walk on the hexagonal lattice, $\mu(1)=\mu(2)=0$. Therefore $\bar{\mu}=0$. Let

$$
\Sigma(1):=\left(\begin{array}{ll}
\sigma_{1,1} & \sigma_{1,2} \\
\sigma_{2,1} & \sigma_{2,2}
\end{array}\right)
$$

Writing $Y_{1}(1):=\left(Y_{1}^{(1)}(1), Y_{1}^{(2)}(1)\right)$, observe that

$$
\sigma_{1,1}=\mathbf{E}\left[\left(Y_{1}^{(1)}(1)\right)^{2}\right] \text { and } \sigma_{2,2}=\mathbf{E}\left[\left(Y_{1}^{(2)}(1)\right)^{2}\right] .
$$

Also,

$$
\sigma_{1,2}=\sigma_{2,1}=\mathbf{E}\left[Y_{1}^{(1)}(1) Y_{1}^{(2)}(1)\right] .
$$

Writing $\omega=\operatorname{Re}(\omega)+i \operatorname{Im}(\omega)$, it is easy to see that

$$
\sigma_{1,1}=\frac{1}{3}\left(1+(\operatorname{Re}(\omega))^{2}+\left(\operatorname{Re}\left(\omega^{2}\right)\right)^{2}\right) .
$$

Since $\operatorname{Re}(\omega)=\operatorname{Re}\left(\omega^{2}\right)$, therefore,

$$
\sigma_{1,1}=\frac{1}{3}\left(1+2(\operatorname{Re}(\omega))^{2}\right) .
$$

Since $\omega=\frac{1}{2}+i \frac{\sqrt{3}}{2}$, therefore, this implies $\sigma_{1,1}=\frac{1}{2}$. Similarly, since $\operatorname{Im}(\omega)=$ $-\operatorname{Im}\left(\omega^{2}\right)$,

$$
\sigma_{2,2}=\frac{1}{3}\left((\operatorname{Im}(\omega))^{2}+\left(\operatorname{Im}\left(\omega^{2}\right)\right)^{2}\right)=\frac{2}{3}(\operatorname{Im}(\omega))^{2}=\frac{1}{2} .
$$

Since, $\operatorname{Re}(\omega)=\operatorname{Re}\left(\omega^{2}\right)$, and $\operatorname{Im}(\omega)=-\operatorname{Im}\left(\omega^{2}\right)$,

$$
\sigma_{1,2}=\sigma_{2,1}=\frac{1}{3}\left(\operatorname{Re}(\omega) \operatorname{Im}(\omega)+\operatorname{Re}\left(\omega^{2}\right) \operatorname{Im}\left(\omega^{2}\right)\right)=0 .
$$

This proves that $\Sigma(1)=\frac{1}{2} \mathbb{I}_{2}$. Similar calculations show that $\Sigma(2)=\frac{1}{2} \mathbb{I}_{2}$. This implies that $\bar{\Sigma}=\frac{1}{2} \Sigma(1)+\frac{1}{2} \Sigma(2)=\frac{1}{2} \mathbb{I}_{2}$. This completes the proof.

\section{Conclusion}

We have presented in this paper a new method for studying finite or infinite color balanced urn schemes through their representation in the associated Markov chain. It turns out that for any general balanced urn scheme, the sequence of observed colors is a realization of a branching Markov chain with a modified kernel on the random recursive tree. We have shown using such representation that under fairly general conditions one can derive asymptotic of various urn schemes, which otherwise may be very difficult to find. As illustrated by several examples, essentially the asymptotic may be derived if the underlying Markov chain has a proper scaling limit. We believe that this novel approach will provide a better understanding of these new type of urn schemes. 
Remark: While preparing the manuscript we were informed by Cécile Mailler and Jean-François Marckert that they are also working on similar problems. We are happy to note this development and we hope that more attention will be given in studying infinite color urn schemes.

\section{ACKNOWLEDGEMENT}

The authors are grateful to Krishanu Maulik and Codina Cotar for various discussions they had with them at various time points. We are also thankful to Tatyana Turova, whose comments helped us to fix a glitch, which we had in an earlier version of the paper.

\section{REFERENCES}

[1] Krishna B. Athreya and Samuel Karlin. Embedding of urn schemes into continuous time Markov branching processes and related limit theorems. Ann. Math. Statist., 39:1801-1817, 1968.

[2] A. Bagchi and A. K. Pal. Asymptotic normality in the generalized PólyaEggenberger urn model, with an application to computer data structures. SIAM J. Algebraic Discrete Methods, 6(3):394-405, 1985.

[3] Zhi-Dong Bai and Feifang Hu. Asymptotics in randomized urn models. Ann. Appl. Probab., 15(1B):914-940, 2005.

[4] Antar Bandyopadhyay and Debleena Thacker. Rate of convergence and large deviation for the infinite color Pólya urn schemes. Statist. Probab. Lett., 92:232-240, 2014.

[5] Antar Bandyopadhyay and Debleena Thacker. Pólya urn schemes with infinitely many colors. (accepted for publication in Bernoulli Journal, available from http://arxiv.org/pdf/1303.7374v2.pdf), 2016.

[6] Patrick Billingsley. Probability and measure. Wiley Series in Probability and Mathematical Statistics. John Wiley \& Sons Inc., New York, third edition, 1995. A Wiley-Interscience Publication.

[7] David Blackwell and James B. MacQueen. Ferguson distributions via Pólya urn schemes. Ann. Statist., 1:353-355, 1973.

[8] Arup Bose, Amites Dasgupta, and Krishanu Maulik. Multicolor urn models with with reducible replacement matrices. Bernoulli, 15(1):279-295, 2009.

[9] Arup Bose, Amites Dasgupta, and Krishanu Maulik. Strong laws for balanced triangular urns. J. Appl. Probab., 46(2):571-584, 2009.

[10] N. Bourbaki. Éléments de mathématique. Topologie générale. Chapitres 1 à 4 . Hermann, Paris, 1971.

[11] May-Ru Chen, Shoou-Ren Hsiau, and Ting-Hsin Yang. A new two-urn model. J. Appl. Probab., 51(2):590-597, 2014.

[12] May-Ru Chen and Markus Kuba. On generalized Pólya urn models. J. Appl. Probab., 50(4):1169-1186, 2013.

[13] Andrea Collevecchio, Codina Cotar, and Marco LiCalzi. On a preferential attachment and generalized Pólya's urn model. Ann. Appl. Probab., 23(3):12191253, 2013.

[14] Edward Crane, Nicholas Georgiou, Stanislav Volkov, Andrew R. Wade, and Robert J. Waters. The simple harmonic urn. Ann. Probab., 39(6):2119-2177, 2011. 
[15] Charles W. Curtis. Linear algebra. Undergraduate Texts in Mathematics. Springer-Verlag, New York, fourth edition, 1993. An introductory approach.

[16] Amites Dasgupta and Krishanu Maulik. Strong laws for urn models with balanced replacement matrices. Electron. J. Probab., 16:no. 63, 1723-1749, 2011.

[17] Rick Durrett. Probability: theory and examples. Cambridge Series in Statistical and Probabilistic Mathematics. Cambridge University Press, Cambridge, fourth edition, 2010.

[18] Philippe Flajolet, Philippe Dumas, and Vincent Puyhaubert. Some exactly solvable models of urn process theory. In Fourth Colloquium on Mathematics and Computer Science Algorithms, Trees, Combinatorics and Probabilities, Discrete Math. Theor. Comput. Sci. Proc., AG, pages 59-118. Assoc. Discrete Math. Theor. Comput. Sci., Nancy, 2006.

[19] David A. Freedman. Bernard Friedman's urn. Ann. Math. Statist, 36:956-970, 1965.

[20] Bernard Friedman. A simple urn model. Comm. Pure Appl. Math., 2:59-70, 1949.

[21] J. Galambos and E. Seneta. Regularly varying sequences. Proc. Amer. Math. Soc., 41:110-116, 1973.

[22] Raúl Gouet. Strong convergence of proportions in a multicolor Pólya urn. J. Appl. Probab., 34(2):426-435, 1997.

[23] Svante Janson. Functional limit theorems for multitype branching processes and generalized Pólya urns. Stochastic Process. Appl., 110(2):177-245, 2004.

[24] Svante Janson. Limit theorems for triangular urn schemes. Probab. Theory Related Fields, 134(3):417-452, 2006.

[25] Sophie Laruelle and Gilles Pagès. Randomized urn models revisited using stochastic approximation. Ann. Appl. Probab., 23(4):1409-1436, 2013.

[26] Sophie Laruelle and Gilles Pagès. Randomized urn models revisited using stochastic approximation. Ann. Appl. Probab., 23(4):1409-1436, 2013.

[27] Mickaël Launay and Vlada Limic. Generalized Interacting Urn Models. available at http://arxiv.org/abs/1201.3495, 2012.

[28] Robin Pemantle. A time-dependent version of Pólya's urn. J. Theoret. Probab., 3(4):627-637, 1990.

[29] Robin Pemantle. A survey of random processes with reinforcement. Probab. Surv., 4:1-79, 2007.

[30] G. Pólya. Sur quelques points de la théorie des probabilités. Ann. Inst. H. Poincaré, 1(2):117-161, 1930.

[31] Eugene Seneta. Non-negative matrices and Markov chains. Springer Series in Statistics. Springer, New York, 2006. Revised reprint of the second (1981) edition [Springer-Verlag, New York; MR0719544].

(Antar Bandyopadhyay) Theoretical Statistics and Mathematics Unit, Indian StatistiCal Institute, Delhi Centre, 7 S. J. S. Sansanwal Marg, New Delhi 110016, india

Theoretical Statistics and Mathematics Unit, Indian Statistical Institute, Kolkata; 203 B. T. RoAd, Kolkata 700108, INDIA

E-mail address: antar@isid.ac.in

(Debleena Thacker) Mathematical Statistics,, Centre for Mathematical Sciences, Lund University. SE-22100, Lund, Sweden,

E-mail address: thackerdebleena@gmail.com 